\title{
Performance and analysis of saddle point preconditioners for the discrete steady-state Navier-Stokes equations
}

\author{
Howard C. Elman ${ }^{1, \star}$, David J. Silvester ${ }^{2, \star \star}$, Andrew J. Wathen ${ }^{3, \star \star \star}$ \\ 1 Department of Computer Science and Institute for Advanced Computer Studies, \\ University of Maryland, College Park, MD 20742, USA; e-mail: elman@cs.umd.edu \\ 2 Department of Mathematics, University of Manchester, Institute of Science \\ and Technology, Manchester M601QD, UK; e-mail: djs@lanczos.ma.umist.ac.uk \\ 3 Oxford University, Computing Laboratory, Wolfson Building, Parks Road, \\ Oxford OX13QD, UK; e-mail: Andy.Wathen@comlab.ox.ac.uk
}

Received August 5, 2000 / Published online June 20, 2001 - (c) Springer-Verlag 2001

Summary. We examine the convergence characteristics of iterative methods based on a new preconditioning operator for solving the linear systems arising from discretization and linearization of the steady-state NavierStokes equations. With a combination of analytic and empirical results, we study the effects of fundamental parameters on convergence. We demonstrate that the preconditioned problem has an eigenvalue distribution consisting of a tightly clustered set together with a small number of outliers. The structure of these distributions is independent of the discretization mesh size, but the cardinality of the set of outliers increases slowly as the viscosity becomes smaller. These characteristics are directly correlated with the convergence properties of iterative solvers.

Mathematics Subject Classification (1991): 65N22

* This work was supported in part by the National Science Foundation under grant DMS9972490

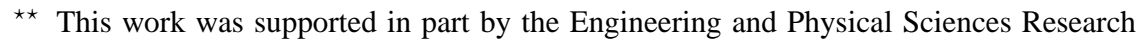
Council Visiting Fellowship Grant GR/N25565

$\star \star \star$ This work was supported in part by the Engineering and Physical Sciences Research Council under grant GR/M59044

Correspondence to: H.C. Elman 


\section{Introduction}

The aim of this work is to study the performance of a preconditioning methodology designed for use with Krylov subspace iteration to compute the numerical solution of the incompressible Navier-Stokes equations. The nonlinear problem is

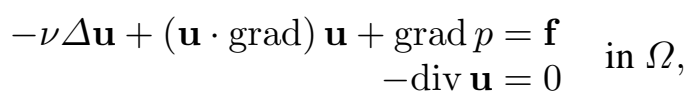

subject to suitable boundary conditions on $\partial \Omega$, where $\Omega$ is an open bounded domain in $\mathbb{R}^{2}$ or $\mathbb{R}^{3}$, and $\mathbf{u}$ and $p$ are the velocity and pressure, respectively. Our focus is on the Oseen equations, the linearized version of (1.1) given by

$$
\begin{aligned}
-\nu \Delta \mathbf{u}+(\mathbf{w} \cdot \operatorname{grad}) \mathbf{u}+\operatorname{grad} p & =\mathbf{f} \\
-\operatorname{div} \mathbf{u} & =0
\end{aligned}
$$

where $\mathbf{w}$ is such that $\operatorname{div} \mathbf{w}=0$. This problem arises for example from a Picard iteration applied to (1.1),

$$
\begin{aligned}
-\nu \Delta \mathbf{u}^{(m)}+\left(\mathbf{u}^{(m-1)} \cdot \operatorname{grad}\right) \mathbf{u}^{(m)}+\operatorname{grad} p^{(m)} & =\mathbf{f} \\
-\operatorname{div} \mathbf{u}^{(m)} & =0
\end{aligned}
$$

where $\mathbf{u}^{(-1)}$ is arbitrary. It is shown in [13] that this iteration is globally convergent provided $\nu>\sqrt{c_{2}\|\mathbf{f}\|_{-1}} / c_{1}$ where $c_{1}$ is the coercivity constant associated with (1.1) and $c_{2}$ is the continuity constant for the convection term; this requirement is also well known as a sufficient condition for the existence of a unique solution to (1.1) [10].

Discretization of (1.2) using a div-stable [10] strategy leads to a linear system of the form

$$
\left(\begin{array}{cc}
F & B^{\mathrm{T}} \\
B & 0
\end{array}\right)\left(\begin{array}{l}
\mathbf{u} \\
p
\end{array}\right)=\left(\begin{array}{l}
\mathbf{f} \\
0
\end{array}\right)
$$

$F$ is a discrete convection-diffusion operator, i.e., it has the form $F=$ $\nu A+N$ where $A$ is a discrete diffusion operator, $N$ is a discrete convection operator, and $B$ and $B^{\mathrm{T}}$ are discrete divergence and gradient operators, respectively.

To motivate a preconditioning strategy for (1.4), we begin with the block LU factorization

$$
\left(\begin{array}{cc}
F & B^{\mathrm{T}} \\
B & 0
\end{array}\right)=\left(\begin{array}{cc}
I & 0 \\
B F^{-1} & I
\end{array}\right)\left(\begin{array}{cc}
F & B^{\mathrm{T}} \\
0 & -B F^{-1} B^{\mathrm{T}}
\end{array}\right) .
$$


With $S=B F^{-1} B^{\mathrm{T}}$ denoting the Schur complement operator, this is equivalent to

$$
\left(\begin{array}{cc}
F & B^{\mathrm{T}} \\
B & 0
\end{array}\right)\left(\begin{array}{ll}
F & B^{\mathrm{T}} \\
0 & -S
\end{array}\right)^{-1}=\left(\begin{array}{cc}
I & 0 \\
B F^{-1} & I
\end{array}\right) .
$$

GMRES iteration [21] applied to a linear system with this coefficient matrix would require precisely two steps to compute the exact solution [16]. A rightoriented preconditioner for (1.4) is determined by approximations $P_{F} \approx F$ and $P_{S} \approx S$, or, more precisely, by operations that approximate the actions of $F^{-1}$ and $S^{-1}$. Variants of this methodology have been considered in many studies $[1,2,4,5,9,15,20,23,25,26]^{1}$.

The advantage of this general approach is that it reduces the problem of finding preconditioners for (1.4) to that of finding good techniques for defining $P_{F}^{-1}$ and $P_{S}^{-1}$. For (1.2), the first subproblem entails approximating the solution of a set of discrete scalar convection-diffusion equations $F u=$ $v . P_{F}^{-1}$ could be defined by applying an iterative algorithm such as multigrid to this system. Our experience is that such an iteration requires only a mild stopping criterion for good performance of the preconditioner, and in this study we will not consider the effects of this approximation and restrict our attention to $P_{F}=F$.

A good choice for $P_{S}$, the approximation to the Schur complement $S$, is less straightforward. Our aim here is to develop an understanding, using a combination of analysis and experiment, of the effectiveness of a new version of this operator developed by Kay and Loghin [14] and considered further by Silvester et. al. [22]. The technique is determined by the choice

$$
P_{S}=A_{p} F_{p}^{-1} M_{p},
$$

where $A_{p}$ and $F_{p}$ represent discrete approximations to scaled Laplacian and convection-diffusion operators defined on the pressure space, and $M_{p}$ is the pressure mass matrix. $P_{S}^{-1}$ was derived from the Green's tensor for the Oseen operator in [14] as an approximation to the inverse of a continuous representation of the Schur complement. We motivate it here by briefly recapitulating an alternative derivation given in [22]. Suppose that there is formal commutativity of differential forms given by

$$
(-\nu \Delta+\mathbf{w} \cdot \nabla) \nabla=\nabla(-\nu \Delta+\mathbf{w} \cdot \nabla)_{p} .
$$

That is, there is a convection-diffusion operator defined on the pressure space (identified with the subscript), and that this operator commutes with the gradient operator as indicated. A discrete version of this relation is

$$
\left(M_{u}^{-1} F\right)\left(M_{u}^{-1} B^{\mathrm{T}}\right)=\left(M_{u}^{-1} B^{\mathrm{T}}\right)\left(M_{p}^{-1} F_{p}\right),
$$

\footnotetext{
${ }^{1}$ Right orientation is largely an arbitrary choice; a left-oriented approach can be derived by a similar argument. For symmetric problems such as the Stokes equations, a block-diagonal preconditioner is preferable [7]
} 
where $M_{u}$ is the mass matrix on the velocity space. This is equivalent to

$$
F^{-1} B^{\mathrm{T}}=M_{u}^{-1} B^{\mathrm{T}} F_{p}^{-1} M_{p} .
$$

Recognizing (1.6)-(1.7) as formal approximations rather than identities and premultiplying (1.7) by $B$ gives the approximation $S \approx P_{S}$ of (1.5), where $A_{p}=B M_{u}^{-1} B^{\mathrm{T}}$. More generally, $A_{p}$ can be any stable discretization of the Poisson operator on the pressure space. This approach for preconditioning represents an improvement and simplification of related strategies developed previously in $[4,5]$.

The boundary conditions associated with the discrete operators $A_{p}$ and $F_{p}$ of (1.5) need to be appropriately defined. For the common case of an enclosed flow with specified velocity conditions on $\partial \Omega$, the discrete Schur complement operator $B F^{-1} B^{\mathrm{T}}$ is conventionally associated with a Neumann operator for the pressure field, see [22]. This means that $A_{p}$ and $F_{p}$ should correspond to discrete elliptic problems with a standard Neumann boundary condition. This issue is explored more fully in [25, pp. 50-51] and [3, pp. 36-43]. In the case of a boundary segment with standard outflow boundary conditions, the Schur complement $S$ (and its preconditioner $P_{S}$ ) must be defined with Dirichlet data for the pressure on that part of the boundary in order to ensure that the preconditioning operator is elliptic over the pressure solution space.

In this paper we examine the properties of this preconditioner. In Sect. 2, we define a set of benchmark problems derived from the "leaky" lid-driven cavity problem, and we study the performance of the preconditioned GMRES iterative solver applied to them. Our concerns here are to understand the dependence on fundamental parameters in the problems, the viscosity $\nu$ and the mesh size $h$ associated with the discretization. Our observations are that the number of iterations required for convergence is independent of the mesh size, but there is some dependence on the viscosity. This dependence is manifested through a period of latency in the early stages of the iteration whose length is mildly increasing with $\nu^{-1}$; after this a faster asymptotic convergence rate is obtained. (We will also occasionally state our observations in terms of the dimensionless Reynolds number $R e=\frac{\mathbf{u} L}{\nu}$, where in our examples the length scale and velocity scales are $L=1,|\mathbf{u}|=1$, so that $R e$ is essentially the same as $\nu^{-1}$.) In Sect. 3, we study the eigenvalues of the preconditioned operator $S P_{S}^{-1}$ for these benchmark problems. We demonstrate empirically that there is a tightly clustered set of eigenvalues contained in a region whose boundaries are independent of $\nu$ and $h$, together with a very small number (on the order of ten) of outlying values that are independent of $h$ but not $\nu$. In Sect. 4, using an analysis of optimal Krylov subspace methods, we give a theoretical explanation of the convergence behavior of GMRES: the latency in iterations is caused by the outlying eigenvalues, and 
the subsequent rapid convergence is a consequence of the tight clustering of the majority of eigenvalues. In Sect. 5, we consider a variant of the problem studied in the previous sections and present analytic bounds on eigenvalue distributions that identify asymptotic effects in the cases of small $\nu$ (large $R e$ ) and large $\nu$ (the Stokes limit). In Sect. 6, we show that our results, which are derived for a fixed velocity field $\mathbf{w}$ in (1.2), are consistent with the performance for a complete Picard iteration (1.3). Finally, in Sect. 7, we make some concluding remarks.

\section{Performance of GMRES iteration}

As a source of benchmark problems, we use a linearized version of the driven cavity problem, in which (1.2) is posed on $\Omega=(0,1) \times(0,1)$, with $\mathbf{f}=0$, boundary conditions

$$
\left\{\begin{array}{l}
u_{1}=u_{2}=0 \quad \text { for } x=0, x=1 \text { or } y=0, \\
u_{1}=1, u_{2}=0 \text { for } y=1,
\end{array}\right.
$$

and convection coefficients (or "wind")

$$
\mathbf{w}=\left(\begin{array}{c}
2(2 y-1)\left(1-(2 x-1)^{2}\right) \\
-2(2 x-1)\left(1-(2 y-1)^{2}\right)
\end{array}\right) .
$$

This choice of the wind, which contains a single recirculation in $\Omega$, is used to simulate what happens in the Picard iteration (1.3) for the driven cavity problem, where $\mathbf{u}^{(m-1)}$ contains one primary recirculation for most $m$; we will return to this point in Sect. 6. We examine two discretizations, the marker-and-cell (MAC) finite difference scheme [11], and the $P_{2}-P_{1}$ finite element discretization, which uses triangular elements with piecewise quadratic bases for the velocity components and a piecewise linear basis for the pressure. Both these discretizations are known to be div-stable, see [10, 17]. For each of them, we discretize (1.2) in $\Omega$ using a uniform mesh of width $h$. (Cf. [14] for results on non-isotropic grids.) The MAC scheme is formally first order accurate when measured in a discrete $H^{1}$ norm, see [17, 18]. The $P_{2}-P_{1}$ method is a higher order discretization: if the weak solution of (1.1) is sufficiently regular, then the velocity converges like $O\left(h^{2}\right)$ when measured in the $H^{1}$ norm, see [10, pp. 181, 323]. $A_{p}$ and $F_{p}$ are defined on the discrete pressure spaces in the natural way via finite differences for MAC and linear elements for $P_{2}-P_{1}$.

We present two sets of experimental results on convergence. These and all other computations described in the paper were performed using Matlab on a Sun Sparc Ultra 1 computer. Table 2.1 shows the number of iterations required by the preconditioned GMRES solver, for both discretizations and 

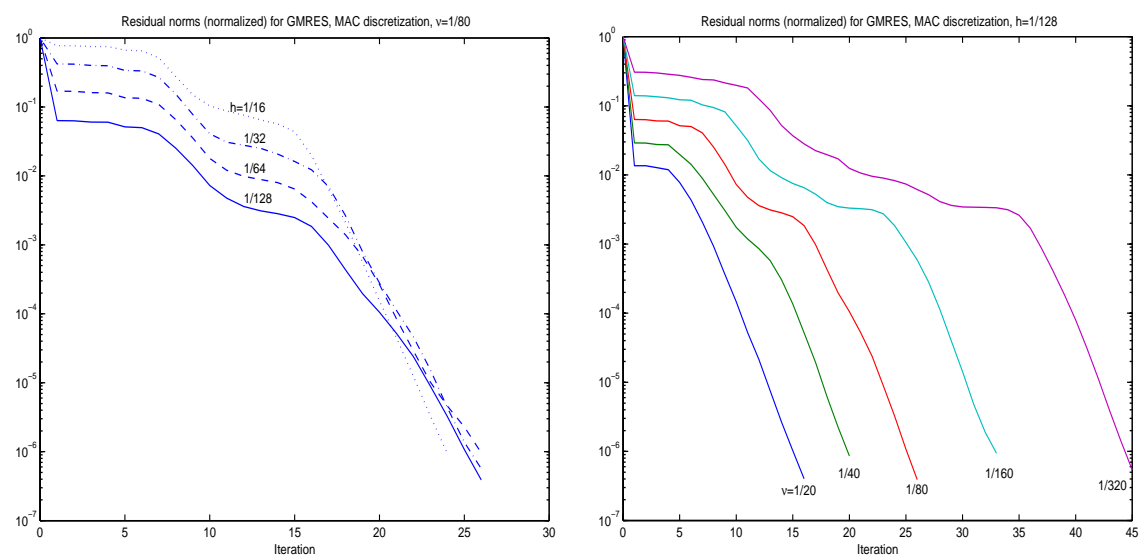

Fig. 2.1. Complete record of $\left\|r_{k}\right\|_{2} /\left\|r_{0}\right\|_{2}$ generated by GMRES, for finite differences and $\nu=1 / 80$ (left) and $h=1 / 128$ (right)

Table 2.1. Iteration counts for convergence of the preconditioned GMRES solver

\begin{tabular}{rccccc}
\hline \multicolumn{6}{c}{ Mac finite differences } \\
\hline \multicolumn{7}{c}{$\nu$} \\
& $1 / 20$ & $1 / 40$ & $1 / 80$ & $1 / 160$ & $1 / 320$ \\
\hline$h=1 / 16$ & 17 & 20 & 24 & 31 & 39 \\
$1 / 32$ & 17 & 21 & 26 & 33 & 43 \\
$1 / 64$ & 16 & 21 & 26 & 34 & 44 \\
$1 / 128$ & 16 & 20 & 26 & 33 & 45 \\
\hline \multicolumn{6}{c}{ finite elements } \\
\hline \multicolumn{6}{c}{$\nu-P_{1}$} \\
\hline$h=1 / 16$ & 18 & 21 & 26 & 37 & 62 \\
$1 / 32$ & 17 & 21 & 26 & 33 & 47 \\
$1 / 64$ & 17 & 20 & 25 & 33 & 43 \\
\hline \multicolumn{7}{c}{}
\end{tabular}

a variety of values of the mesh size $h$ and viscosity parameter $\nu$. The initial guess was identically zero, and the stopping criterion was

$$
\frac{\left\|r_{k}\right\|_{2}}{\left\|r_{0}\right\|_{2}}<10^{-6}
$$

where $r_{k}$ is the residual vector. Figures 2.1 and 2.2 show the details of convergence histories for some of these entries. The left sides of the figures show the results for fixed values of the viscosity parameter $(\nu=1 / 80)$ and several mesh sizes, and the right sides show the results for fixed values of the mesh size $(h=1 / 128$ for the MAC discretization and $h=1 / 64$ for $\left.P_{2}-P_{1}\right)$ and several values of $\nu$. 

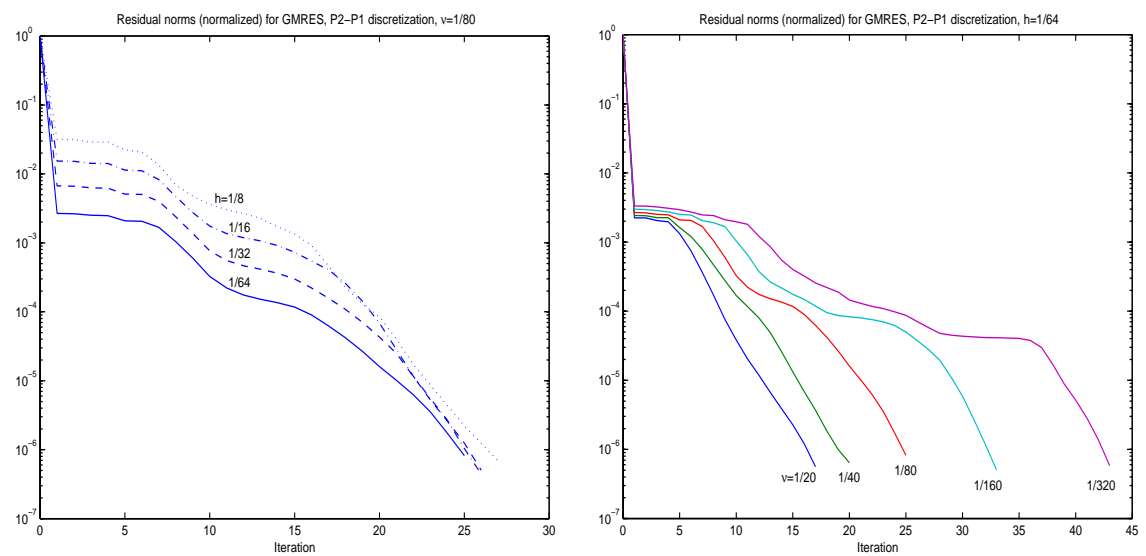

Fig. 2.2. Complete record of $\left\|r_{k}\right\|_{2} /\left\|r_{0}\right\|_{2}$ generated by GMRES, for $P_{2}-P_{1}$ finite elements and $\nu=1 / 80$ (left) and $h=1 / 64$ (right)

These experiments clearly show that convergence is essentially independent of the discretization parameter but that there is modest dependence on the viscosity, with reductions in $\nu$ (i.e., increases in the Reynolds number) leading to increases in iteration counts. The tabulated results are consistent with those obtained by Kay and Loghin [14] for this problem as well as two others, models of a pipe-flow and flow over a backward facing step. The figures suggest that in fact the asymptotic convergence behavior of the GMRES iteration is also independent of $\nu$, but that there is a period of slow convergence in the early stages of the iteration, and this latency period is longer for smaller values of $\nu$. These observations hold for both discretizations.

\section{Behavior of eigenvalues}

Let the preconditioned system under consideration be denoted now by

$$
\mathcal{A} x=f,
$$

and let the residual associated with an iterate $\boldsymbol{x}_{m}$ be given by

$$
\boldsymbol{r}_{m}=\boldsymbol{f}-\mathcal{A} \boldsymbol{x}_{m} .
$$

Assume $\mathcal{A}=V \Lambda V^{-1}$ is diagonalizable, and let $\sigma(\mathcal{A})$ denote the set of eigenvalues of $\mathcal{A}$. Recall the standard bound on convergence of GMRES [21],

$$
\left\|\boldsymbol{r}_{m}\right\|_{2} \leq \kappa(V) \min _{p_{m}(0)=1} \max _{\lambda \in \sigma(\mathcal{A})}\left|p_{m}(\lambda)\right|\left\|\boldsymbol{r}_{0}\right\|_{2}
$$




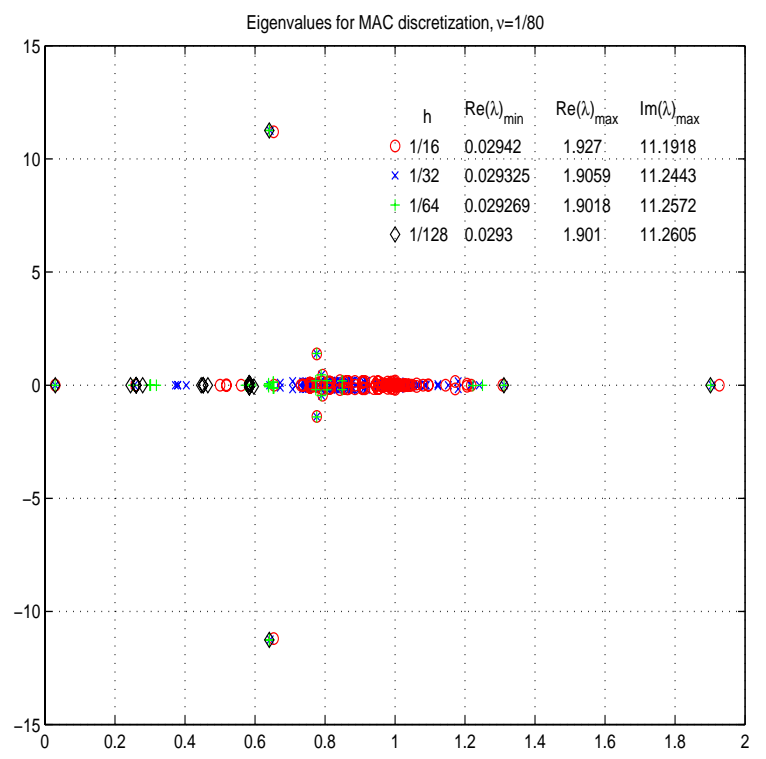

Fig. 3.1. Dependence of eigenvalues on mesh size for the MAC discretization, $\nu=1 / 80$

where the minimum is over all polynomials of degree $m$ taking on the value 1 at the origin. In this section, we show the results of a series of experiments concerning the eigenvalues of the $F_{p}$-preconditioned matrices $S P_{S}^{-1}$ for the benchmark problems considered above. We will then (in Sect. 4) use these results together with (3.2) to explain convergence performance.

We begin with the MAC discretization. Figure 3.1 plots the eigenvalues of the preconditioned matrices, for $\nu=1 / 80$ and four successively refined meshes. For $h=1 / 16$ and $1 / 32$, all the eigenvalues are displayed, and for $h=1 / 64$ and $1 / 128$, a subset is shown consisting of those with smallest nonzero real parts, largest real parts, and largest imaginary parts ${ }^{2}$. Figure 3.2 shows the extremal eigenvalues for three values of $\nu$ with $h=1 / 64$.

These figures give a clear picture of the dependence of the large extremal eigenvalues on both the mesh size $h$ and viscosity $\nu$, as well as the dependence of the smallest eigenvalues on the mesh size. Figure 3.1 shows that the extremal eigenvalues display no significant dependence on $h$; indeed, the tabulated results included in the figure show that the maximum real parts, maximum imaginary parts, and minimum real parts of all eigenvalues are

\footnotetext{
${ }^{2}$ For $h=1 / 64$, this set consists of the nineteen eigenvalues with smallest nonzero real parts, the four with largest real parts and the six pairs with largest imaginary parts. For $h=1 / 128$, it is the nineteen with smallest real part, the two with largest real parts, and the single pair with largest imaginary part. The zero eigenvalue corresponding to hydrostatic pressure is excluded from these considerations. The computations were done with the Matlab sparse eigenvalue routine eigs
} 


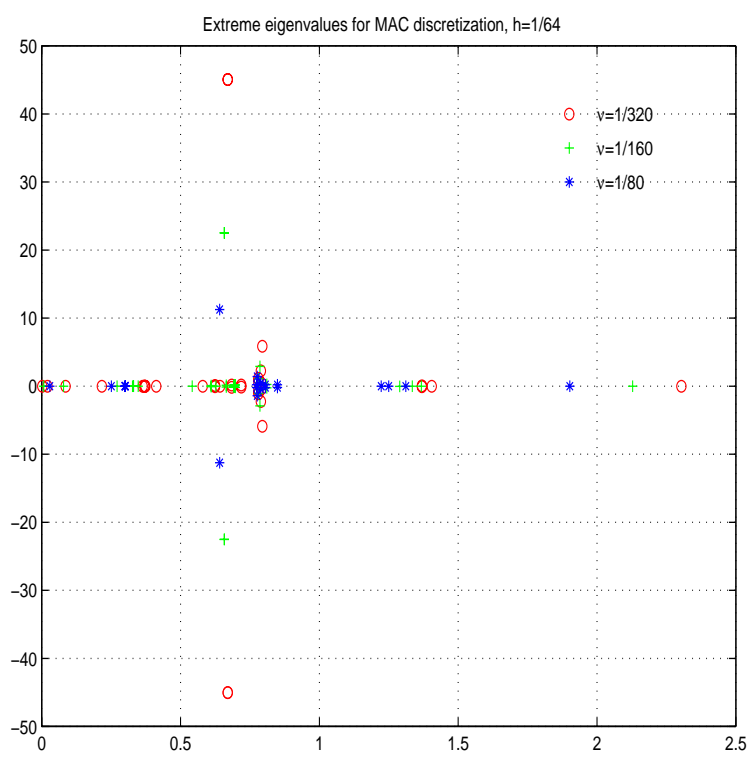

Fig. 3.2. Dependence of eigenvalues on viscosity for the MAC discretization, $h=1 / 64$

Table 3.1. Dependence of eigenvalues on $\nu$ for the MAC discretization and $h=1 / 128$

\begin{tabular}{lcccccc}
\hline \multicolumn{4}{c}{ Extremal real and } \\
imaginary parts & \multicolumn{3}{c}{ Other small real parts } \\
$\nu$ & $\Re(\lambda)_{\min }$ & $\Re(\lambda)_{\max }$ & $\Im(\lambda)_{\max }$ & $\lambda_{2}$ & $\lambda_{3}$ & $\lambda_{4}$ \\
\hline $1 / 20$ & .2402 & 1.2017 & 2.8015 & .2410 & .2411 & .3323 \\
$1 / 40$ & .1073 & 1.5389 & 5.6224 & .2486 & .2495 & .2553 \\
$1 / 80$ & .0293 & 1.9010 & 11.2605 & .2444 & .2597 & .2608 \\
$1 / 160$ & .0075 & 2.1265 & 22.5330 & .0786 & .2635 & .2747 \\
$1 / 320$ & .0019 & 2.3008 & 45.0749 & .0209 & .0860 & .2158 \\
$1 / 640$ & .0005 & 2.4403 & 90.1561 & .0053 & .0230 & .1388 \\
\hline
\end{tabular}

virtually identical for all four mesh parameters. Figure 3.2 shows that the maximal real parts in the eigenvalue sets are also close to being constant as functions of $\nu$, but that the maximal imaginary parts grow like $\nu^{-1}$. The dependence of the small real parts on $\nu$ is less clear from this figure. This is explored further in Table 3.1, which shows the extreme real and imaginary parts, as well as the three next smallest real parts, in this case for $h=1 / 128$. The patterns are as follows. For small enough $\nu$, the smallest real part is actually decreasing in proportion to $\nu^{2}$. A similar statement applies for the other small real parts, although the asymptotic behavior is evident only for somewhat smaller values of $\nu$. That is, there are some eigenvalues that are decreasing in proportion to $\nu^{2}$, but the set (of indices of eigenvalues) for which this pattern holds appears to be small. 
Table 3.2. Number of eigenvalues outside circle, for MAC discretization and $h=1 / 16$, $h=1 / 32$

\begin{tabular}{lcccc}
\hline & \multicolumn{2}{c}{$\begin{array}{c}\text { No. eigenvalues } \\
\text { outside circle }\end{array}$} & \multicolumn{2}{c}{$\begin{array}{c}\text { No. real eigs } \\
\text { left of circle }\end{array}$} \\
$\nu$ & $1 / 16$ & $1 / 32$ & $1 / 16$ & $1 / 32$ \\
\hline $1 / 40$ & 7 & 7 & 1 & 1 \\
$1 / 80$ & 8 & 8 & 2 & 2 \\
$1 / 160$ & 11 & 12 & 3 & 3 \\
$1 / 320$ & 19 & 21 & 4 & 4 \\
$1 / 640$ & 25 & 29 & 6 & 6 \\
\hline
\end{tabular}

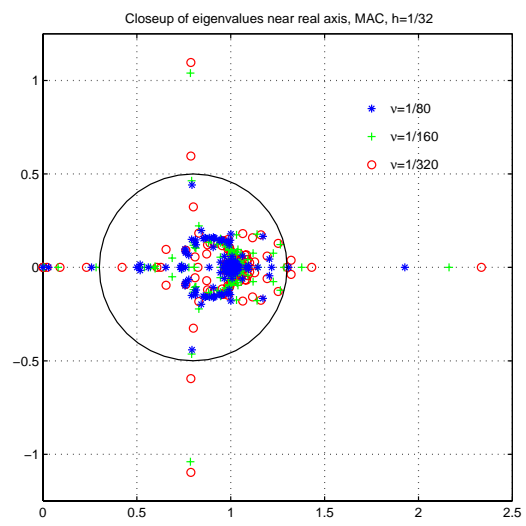

Circle, center 8 radius .5

Fig. 3.3. Containment of eigenvalues in a clustered set, MAC discretization, $h=1 / 32$

Figures 3.1 and 3.2 also suggest that with the exception of a small set of outliers of the types given above, the majority of eigenvalues lie in a tightly clustered set. This issue is examined in Fig. 3.3, which shows a magnified image of the data for $h=1 / 32$ from Fig. 3.2 (coordinates on the imaginary axis are restricted), on which a circle centered at $(0.8,0)$ with radius $1 / 2$ is drawn. The circle represents a domain, determined by inspection, containing the majority of eigenvalues. Table 3.2 continues in this direction by showing how many eigenvalues lie outside this circle for different values of $\nu$, for the two mesh parameters for which all eigenvalues have been computed. These data indicate that in fact the number of outliers is small but growing slightly with $\nu^{-1}$. The number of eigenvalues with small real parts (i.e., to the left of the circle) is also increasing as $\nu$ gets smaller, but quite a bit more slowly than the total number of outliers.

We next discuss an analogous set of computations for the $P_{2}-P_{1}$ discretization. Table 3.3 shows a set of extremal eigenvalues of the preconditioned matrices, for $h=1 / 64$. This data is analogous to that shown in Table 3.1 for finite differences, and the patterns displayed for the two dis- 
Table 3.3. Dependence of eigenvalues on $\nu$ for the $P_{2}-P_{1}$ discretization and $h=1 / 64$

\begin{tabular}{lcccccc}
\hline \multicolumn{4}{c}{ Extremal real and } \\
imaginary parts & \multicolumn{3}{c}{ Other small real parts } \\
$\nu$ & $\Re(\lambda)_{\min }$ & $\Re(\lambda)_{\max }$ & $\Im(\lambda)_{\max }$ & $\lambda_{2}$ & $\lambda_{3}$ & $\lambda_{4}$ \\
\hline $1 / 20$ & .1336 & 1.2009 & 2.8012 & .2068 & .2069 & .2489 \\
$1 / 40$ & .1072 & 1.5386 & 5.6218 & .1337 & .2091 & .2100 \\
$1 / 80$ & .0293 & 1.8999 & 11.2595 & .1338 & .2114 & .2117 \\
$1 / 160$ & .0075 & 2.1248 & 22.5312 & .0786 & .1339 & .2148 \\
$1 / 320$ & .0019 & 2.2984 & 45.0714 & .0209 & .0859 & .1339 \\
$1 / 640$ & .0005 & 2.4366 & 90.1491 & .0053 & .0230 & .0553 \\
\hline
\end{tabular}

Table 3.4. Eigenvalues for the $P_{2}-P_{1}$ discretization on coarse grids

\begin{tabular}{lcccccc}
\hline \multicolumn{3}{c}{ Extremal real and } \\
imaginary parts & \multicolumn{3}{c}{ Other small real parts } \\
$h=1 / 32$ & $\Re(\lambda)_{\min }$ & $\Re(\lambda)_{\max }$ & $\Im(\lambda)_{\max }$ & $\lambda_{2}$ & $\lambda_{3}$ & $\lambda_{4}$ \\
\hline $1 / 20$ & .1339 & 1.1984 & 2.7996 & .2131 & .2132 & .2825 \\
$1 / 40$ & .1073 & 1.5376 & 5.6188 & .1342 & .2160 & .2174 \\
$1 / 80$ & .0293 & 1.8976 & 11.2534 & .1341 & .2186 & .2193 \\
$1 / 160$ & .0075 & 2.1213 & 22.5190 & .0787 & .1341 & .1718 \\
$1 / 320$ & .0019 & 2.2931 & 45.0470 & .0209 & .0720 & .0727 \\
$1 / 640$ & .0005 & 2.4284 & 90.1004 & .0053 & .0230 & .0292 \\
\hline$h=1 / 16$ & & & & & & \\
$\nu$ & & & & & & \\
\hline $1 / 20$ & .1344 & 1.1890 & 2.7933 & .2215 & .2216 & .3022 \\
$1 / 40$ & .1076 & 1.5334 & 5.6064 & .1349 & .2250 & .2250 \\
$1 / 80$ & .0294 & 1.8880 & 11.2288 & .1345 & .2012 & .2012 \\
$1 / 160$ & .0075 & 2.1062 & 22.4699 & .0792 & .1042 & .1042 \\
$1 / 320$ & .0019 & 2.2684 & 44.9480 & .0211 & .0470 & .0476 \\
$1 / 640$ & .0005 & 2.3864 & 89.8966 & .0054 & .0160 & .0160 \\
\hline
\end{tabular}

cretizations are largely the same. That is, the smallest real parts are of order $\nu^{2}$, the largest real parts are $O(1)$, and the largest imaginary parts proportional to $O\left(\nu^{-1}\right)$.

We study the dependence of spectra on mesh size by tabulating additional data for coarser meshes in Table 3.4. From the results in this table and Table 3.3, it is clear that, just as above, the smallest real parts, largest real parts and largest imaginary parts of eigenvalues are all independent of the mesh size.

For this discretization, it is more difficult to make a clean statement about clustering of the non-extreme eigenvalues. A table analogous to Table 3.3 for the finest mesh for which we can compute a complete set of eigenvalues ( $h=1 / 32$ ) would show many more eigenvalues lying outside a fixed set as $\nu$ is reduced. For example, for $h=1 / 32$ and $\nu=1 / 320$, there are 191 
eigenvalues outside the circle depicted in Fig. 3.3; this contrasts with 21 for the MAC discretization. We do not believe that this is caused by any inadequacy of the preconditioning operator, but that instead it stems from properties of the discretization. This point can be explored using the data in Tables 3.3 and 3.4. As noted above, the extremal eigenvalues are the same for the three mesh parameters of the tables. When $\nu$ is large, the other eigenvalues $\left(\lambda_{2}, \lambda_{3}, \lambda_{4}\right)$ are also qualitatively similar for the three values of $h$. However, as $\nu$ becomes smaller, these eigenvalues behave differently on coarse meshes than on finer ones. For example, for $\nu=1 / 640, \lambda_{3}$ is much smaller for $h=1 / 16$ than for the finer meshes, and a similar statement holds for $\lambda_{4}$ for small values of $\nu$. As $h$ is refined, these eigenvalues actually increase and become closer to the analogous values for the MAC discretization. That is, for small $\nu$ and insufficiently fine meshes, many of the eigenvalues occurring in these computations are artificially small and do not indicate a proper dependence on $\nu$. As an additional example, the bottom right entry .0553 of Table 3.3 is also likely to be artificially small.

We believe the reason for this has to do with the accuracy of the discretization. It is well known that if the cell Reynolds number $R e_{h}=\frac{|\mathbf{w}| h}{2 \nu}$ is large, then the discretization may be inaccurate. The $P_{2}-P_{1}$ discretization has a higher order of accuracy than the finite difference discretization, but it also requires finer meshes for its asymptotic properties to hold; when the mesh is too coarse, the discretization is less accurate and this leads to anomolous behavior in the preconditioning process when $R e_{h}$ is large. This is also the likely explanation for the reduction in iterations seen in Table 2.1 for small $\nu$ as $h$ is reduced.

\section{Analysis of GMRES convergence}

In this section, we derive a convergence bound for GMRES iteration that establishes a connection between the latencies displayed in the initial stages of the solution process, as shown in Sect. 2, and the outlying eigenvalues of the preconditioned systems described in Sect. 3. The analysis is based on a technique developed by Jennings [12] for the conjugate gradient method, whereby a bound is obtained using certain auxiliary polynomials.

With the preconditioned problem represented as in (3.1), suppose as in our case that $\sigma(\mathcal{A})=\sigma_{c}(\mathcal{A}) \cup \sigma_{o}(\mathcal{A})$ where $\sigma_{c}(\mathcal{A})$ denotes a clustered set of eigenvalues of $\mathcal{A}$ and $\sigma_{o}(\mathcal{A})=\left\{\lambda_{1}, \lambda_{2}, \ldots, \lambda_{d}\right\}$ denotes a set of $d$ outliers. Taking $m=d+k$ in (3.2), we have

$$
\min _{p_{k+d}(0)=1} \max _{\lambda \in \sigma(\mathcal{A})}\left|p_{k+d}(\lambda)\right| \leq \max _{\lambda \in \sigma_{c}(\mathcal{A})}\left|\phi_{d}(\lambda)\right|\left|C_{k}(\lambda)\right|,
$$

where

$$
\phi_{d}(\lambda)=\left(1-\frac{1}{\lambda_{1}} \lambda\right)\left(1-\frac{1}{\lambda_{2}} \lambda\right) \cdots\left(1-\frac{1}{\lambda_{d}} \lambda\right)
$$


is the polynomial of degree $d$ whose roots are the outlying eigenvalues contained in $\sigma_{o}(\mathcal{A})$, and $C_{k}$ is any polynomial of degree $k$ satisfying $C_{k}(0)=1$. $C_{k}$ can be chosen to be small on a set $\mathcal{E}$ containing $\sigma_{c}(\mathcal{A})$ such that

$$
\max _{\lambda \in \sigma_{c}(\mathcal{A})}\left|C_{k}(\lambda)\right| \leq \approx \rho^{k} .
$$

If the enclosing set $\mathcal{E}$ is bounded by a circle centered at $c$ with radius $r$ as in Sect. 3, then the choice $C_{k}(\lambda)=\left(\frac{c-\lambda}{c}\right)^{k}$ is optimal with respect to $L_{\infty}$ on $\mathcal{E}[19$, p. 90]. For this choice, $\rho=r / c$, and it follows from (3.2) and (4.1) that

$$
\left\|\boldsymbol{r}_{m}\right\|_{2} \leq \kappa(V) \max _{\lambda \in \sigma_{c}(\mathcal{A})}\left|\phi_{d}(\lambda)\right| \rho^{k}\left\|\boldsymbol{r}_{0}\right\|_{2}
$$

If all the outlying eigenvalues are larger in modulus than the (clustered) members of $\sigma_{c}(\mathcal{A})$, then for any $\lambda \in \sigma_{c}(\mathcal{A}),\left|\frac{\lambda}{\lambda_{j}}\right|<1$ and $\left|\phi_{d}(\lambda)\right|<1$. The bound (4.2) then suggests that there will be a latency of $d$ steps before the asymptotic convergence behavior is observed. If some outlier $\lambda_{j}$ is small, then the factor $\left(1-\frac{1}{\lambda_{j}} \lambda\right)$ of $\phi_{d}$ will be large, and there may be some additional delay. The small eigenvalues identified in Sect. 3 are of magnitude $\nu^{2}$, so that the corresponding factors in $\phi_{d}$ are of order $\nu^{-2}$. If there are $s$ small eigenvalues like this, and (as above) the clustered eigenvalues have magnitude $O(1)$, then the term

$$
\max _{\lambda \in \sigma_{c}(\mathcal{A})}\left|\phi_{d}(\lambda)\right| \rho^{k}
$$

will be bounded by an expression essentially of the form $\left(\frac{1}{\nu^{2}}\right)^{s} \rho^{k}$, and the asymptotic convergence behavior will not be manifested until this expression is less than 1 . This will be achieved when

$$
k>2 s \frac{\log \nu^{-1}}{\log \rho^{-1}} .
$$

That is, for the preconditioned problems under consideration, we expect convergence to be slow for a number of iterations essentially determined by the number of outlying eigenvalues; if some of these eigenvalues are small, then there may be an additional delay of magnitude $\log \nu^{-1}$. In our examples (Table 3.2), there are fewer small outliers than large ones, and therefore this extra delay should be relatively short.

We next show that the bounds established with this analysis give an accurate depiction of performance. Let $p_{m}$ now denote the iteration polynomial generated by $m$ steps of the GMRES iteration. The roots of $p_{m}$, which are referred to as the harmonic Ritz values, are the eigenvalues of the matrix

$$
\left(\tilde{H}_{m}^{*}\right)^{-1}\left(H_{m}^{*} H_{m}\right)
$$




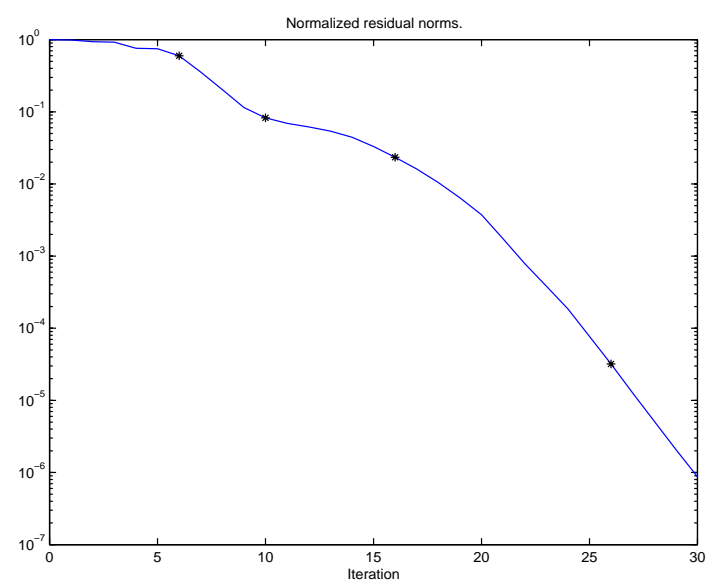

Fig. 4.1. Convergence history of GMRES for the preconditioned Schur complement system with $\nu=1 / 80, h=1 / 32, P_{2}-P_{1}$ discretization, and steps $6,10,16$ and 26 highlighted

(see [8]), where $H_{m}$ is the rectangular upper Hessenberg matrix of dimensions $(m+1) \times m$ generated by the Arnoldi computation used for GMRES [21], and $\tilde{H}_{m}$ is the square submatrix of $H_{m}$ obtained by removing the last row. As the iteration converges to the solution, the harmonic Ritz values converge to eigenvalues of the coefficient matrix.

For simplicity, we consider here the GMRES iteration applied directly to the Schur complement system $S p=s$ where $s=B F^{-1} f$, with right preconditioner $P_{S}$. We present results for the $P_{2}-P_{1}$ discretization, with $\nu=1 / 80$ and $h=1 / 32$. Figure 4.1 shows the convergence history, with values of the residual norms at four distinguished stages of the iteration highlighted with asterisks. Figures 4.2-4.5 depict the state of the GMRES iteration at the four stages. The figures show the eigenvalues of $S P_{S}^{-1}$, together with the $m$ harmonic Ritz values available at step $m$, for $m=6$, 10,16 and 26. The left sides of the figures show the complete set of data; the right sides show magnified views by limiting the dimensions on the imaginary axis.

Some of the trends displayed in these figures are as follows. Step 6 (Fig. 4.2) is the first where any eigenvalues of the coefficient matrix, in this case the pair with maximal imaginary parts, are accurately resolved. At this stage, the four other harmonic Ritz values bear no clear relation to the spectrum of $S P_{S}^{-1}$. Also, after this step, the residual norm exhibits a noticeable decrease in size (Fig. 4.1). At step 10 (Fig. 4.3), several other extremal eigenvalues are resolved: the pair with second largest imaginary part and the one with largest real part. In addition, the estimates for the pair with third largest imaginary part are fairly accurate, although it takes until step 14 for these to be resolved well. In these first two figures, there is no 

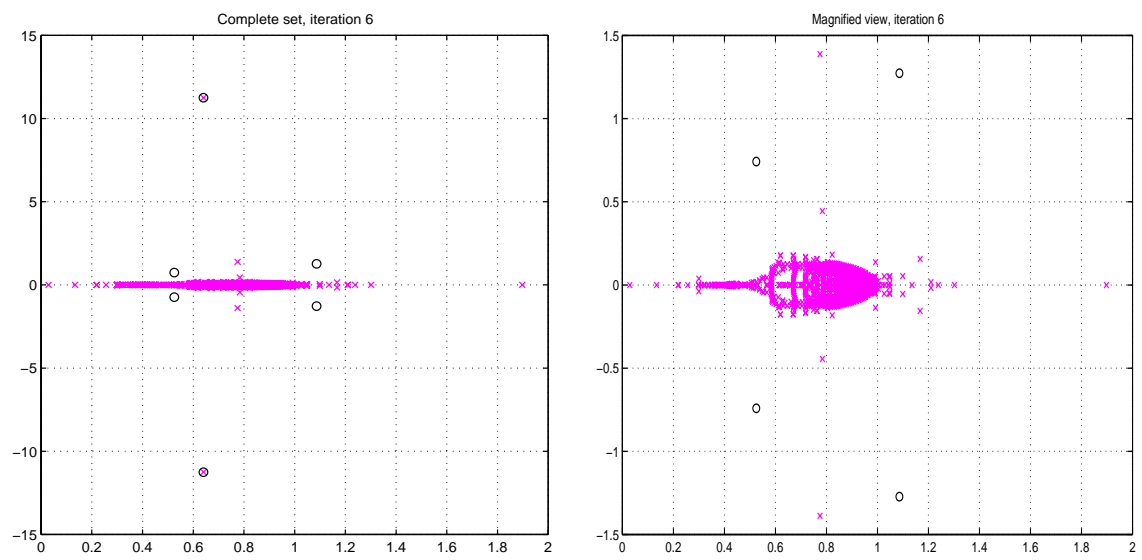

Fig. 4.2. Eigenvalues $(\times)$ and harmonic Ritz values $(\circ)$ at GMRES step 6 for the preconditioned Schur complement system, with $\nu=1 / 80, h=1 / 32, P_{2}-P_{1}$ discretization
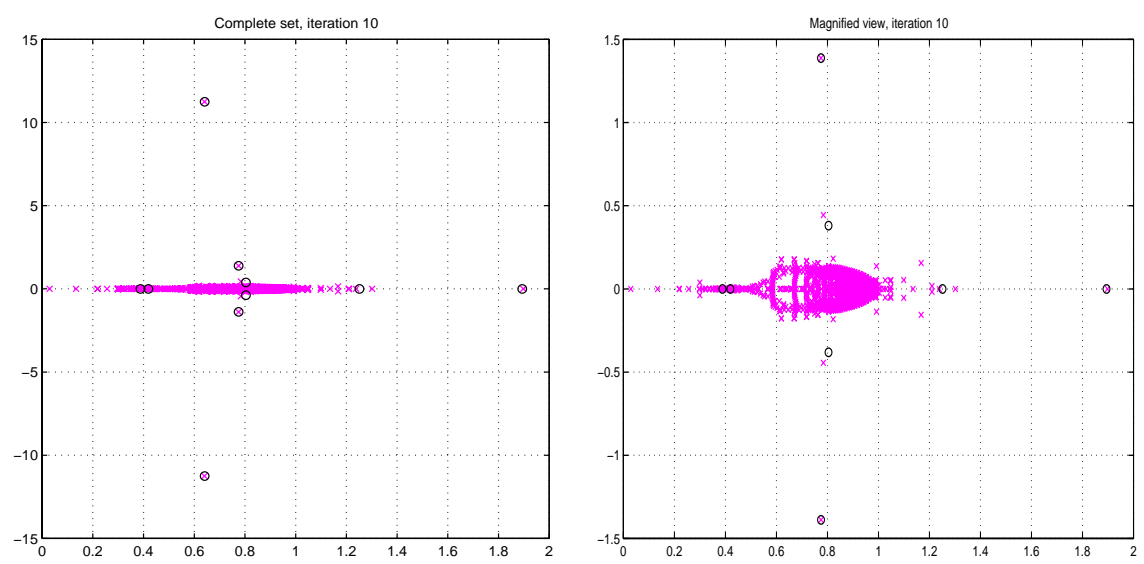

Fig. 4.3. Eigenvalues $(\times)$ and harmonic Ritz values (o) at GMRES step 10 for the preconditioned Schur complement system, with $\nu=1 / 80, h=1 / 32, P_{2}-P_{1}$ discretization

harmonic Ritz value near the eigenvalue with smallest real part. By step 16 (Fig. 4.4), the smallest harmonic Ritz value is close to that eigenvalue; good resolution is obtained at step 18. At about this point, the GMRES iteration enters its period of asymptotic performance. Finally, at step 26, when the asymptotic performance is established, all extreme eigenvalues are wellapproximated and the new harmonic Ritz estimates lie around the perimeter of the set of eigenvalues clustered in the vicinity of $(0.8,0)$.

These results are typical of performance for both discretizations. They indicate, as the analysis suggests, that asymptotic convergence occurs after the outlying eigenvalues are resolved by the GMRES iteration, and that the smaller outliers take longer to be resolved than the longer ones. Once the 

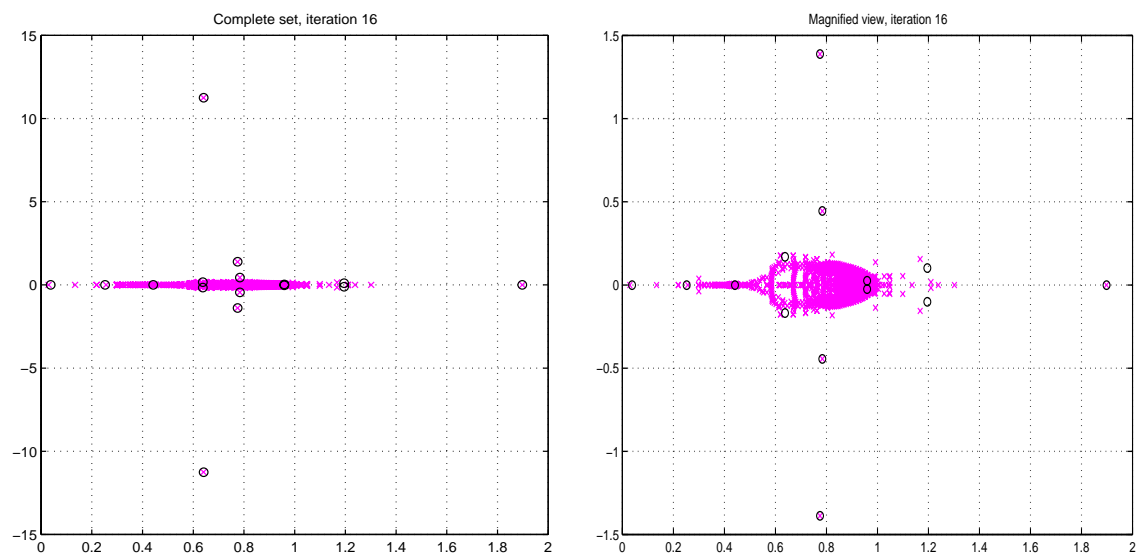

Fig. 4.4. Eigenvalues $(\times)$ and harmonic Ritz values $(\circ)$ at GMRES step 16 for the preconditioned Schur complement system, with $\nu=1 / 80, h=1 / 32, P_{2}-P_{1}$ discretization
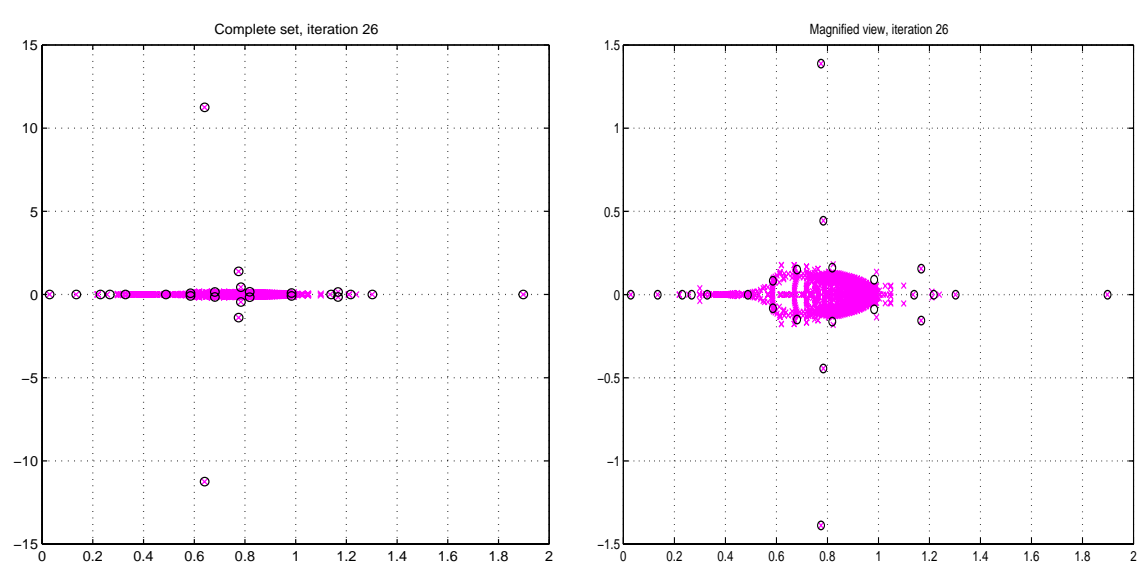

Fig. 4.5. Eigenvalues $(\times)$ and harmonic Ritz values (o) at GMRES step 26 for the preconditioned Schur complement system, with $\nu=1 / 80, h=1 / 32, P_{2}-P_{1}$ discretization

outliers are obtained, the fact that subsequent eigenvalue estimates tend to embrace the cluster is a consequence of the maximum principle.

Remark. The bound (3.2) also depends on the condition number of the matrix of eigenvectors. Using Matlab, we calculated this quantity for the Euclidean norm and various choices of $h$ and $\nu$. It had values on the order of $10^{2}$ to $10^{3}$ but displayed no discernable pattern. The correlation between performance and analysis suggests that the eigenvalues are responsible for convergence behavior, but we are unable to incorporate $\kappa(V)$ into the discussion. 


\section{Analysis of eigenvalues}

In this section, we derive bounds on the eigenvalues of a generalized eigenvalue problem

$$
B F^{-1} B^{\mathrm{T}} p=\mu \hat{P}_{S} p
$$

Here $\hat{P}_{S}$ is a variant of the preconditioner $P_{S}$ of (1.5) but with a "symmetric style,"

$$
\hat{P}_{S}=M_{p}^{1 / 2} A_{p}^{1 / 2} F_{p}^{-1} A_{p}^{1 / 2} M_{p}^{1 / 2} .
$$

The presence of the matrix square roots makes this operator unsuitable for practical computation, but its symmetric form is needed for an analysis. We consider two cases separately. We first show that for the case of small Reynolds numbers (large $\nu$ ), the eigenvalues of (5.1) are perturbations of those of a generalized problem associated with the Stokes equations, which leads to bounds showing that (5.1) is well behaved and that the properties of the preconditioner in the Stokes limit are good. For more general situations, including the case of small $\nu$, we derive an equivalence between (5.1) and an alternative problem that leads to rigorous bounds on the imaginary parts of the eigenvalues and heuristic insight into properties of the real parts.

\subsection{Small Reynolds numbers and the Stokes limit}

Let

$$
F=\nu A+N, \quad F_{p}=\nu A_{p}+N_{p},
$$

where

$$
A=\frac{1}{\nu}\left(\frac{F+F^{\mathrm{T}}}{2}\right), \quad A_{p}=\frac{1}{\nu}\left(\frac{F_{p}+F_{p}^{\mathrm{T}}}{2}\right)
$$

denote the (scaled) symmetric parts of $F$ and $F_{p}$, respectively, and

$$
N=\frac{F-F^{\mathrm{T}}}{2}, \quad N_{p}=\frac{F_{p}-F_{p}^{\mathrm{T}}}{2}
$$

denote the skew-symmetric parts. Consider the factorizations of the discrete convection-diffusion operators,

$$
\begin{aligned}
& F=\nu A+N=\nu A^{1 / 2}\left(I+\frac{1}{\nu} \hat{N}\right) A^{1 / 2} \\
& F_{p}=\nu A_{p}+N_{p}=\nu A_{p}^{1 / 2}\left(I+\frac{1}{\nu} \hat{N}_{p}\right) A_{p}^{1 / 2},
\end{aligned}
$$

where $\hat{N}=A^{-1 / 2} N A^{-1 / 2}, \hat{N}_{p}=A_{p}^{-1 / 2} N_{p} A_{p}^{-1 / 2}$. (Here and in the following, $A_{p}^{-1}$ is to be understood as the inverse of the operator defined on 
the orthogonal complement of the null vector $p \equiv$ constant, or, equivalently, as the pseudo-inverse on the complete pressure space.) It is well known that the spectral radii $\rho(\hat{N})$ and $\rho\left(\hat{N}_{p}\right)$ are bounded by constants that are independent of the mesh parameter $h$ [6]; these spectral radii are clearly also independent of $\nu$. We will use these observations to derive simplified representations of the Schur complement and preconditioning operators for large $\nu$. The following result will be used in the derivation; the proof is straightforward and is omitted.

Lemma 5.1. For any matrix $C$ such that $I+C$ is nonsingular,

$$
(I+C)^{-1}=I-C+C^{2}(I+C)^{-1} .
$$

Since $\hat{N}$ is skew-symmetric, $I+\frac{1}{\nu} \hat{N}$ is nonsingular, and the lemma implies that

$$
\left(I+\frac{1}{\nu} \hat{N}\right)^{-1}=I-\frac{1}{\nu} \hat{N}+\frac{1}{\nu^{2}} E,
$$

where $E=\hat{N}^{2}\left(I+\frac{1}{\nu} \hat{N}\right)^{-1}$. Combining this with the first relation of (5.3) gives the following expression for the Schur complement:

$$
\begin{aligned}
S & =B F^{-1} B^{\mathrm{T}} \\
& =\frac{1}{\nu}\left(S_{\infty}-\frac{1}{\nu} B A^{-1 / 2} \hat{N} A^{-1 / 2} B^{\mathrm{T}}+\frac{1}{\nu^{2}} B A^{-1 / 2} E A^{-1 / 2} B^{\mathrm{T}}\right),
\end{aligned}
$$

where $S_{\infty}=B A^{-1} B^{\mathrm{T}}$ is the Schur complement associated with the Stokes operator. Note also that

$$
\|E\|_{2} \leq \rho(\hat{N})^{2}=O(1) .
$$

An identical argument for $\hat{P}_{S}$ of (5.2) shows that

$$
\left(I+\frac{1}{\nu} \hat{N}_{p}\right)^{-1}=I-\frac{1}{\nu} \hat{N}_{p}+\frac{1}{\nu^{2}} E_{p}
$$

where

$$
E_{p}=\hat{N}_{p}^{2}\left(I+\frac{1}{\nu} \hat{N}_{p}\right)^{-1}, \quad\left\|E_{p}\right\|_{2} \leq \rho\left(\hat{N}_{p}\right)^{2} .
$$

Consequently, the preconditioning operator has the form

$$
\hat{P}_{S}=\frac{1}{\nu}\left(M_{p}-\frac{1}{\nu} M_{p}^{1 / 2} \hat{N}_{p} M_{p}^{1 / 2}+\frac{1}{\nu^{2}} M_{p}^{1 / 2} E_{p} M_{p}^{1 / 2}\right) .
$$

In essence, these derivations quantify the facts that for small Reynolds number, $S$ and $\hat{P}_{S}$ are perturbations of $\frac{1}{\nu} S_{\infty}$ and $\frac{1}{\nu} M_{p}$, respectively. But the eigenvalues for the generalized problem

$$
S_{\infty} p=\eta M_{p} p
$$


are contained in a mesh independent interval $\left[\gamma^{2}, \Gamma^{2}\right]$. Here, $\gamma$ is the infsup constant associated with the discretization, and $\Gamma=1$ for conforming finite element approximations such as $P_{2}-P_{1}$, see [24]. The following result shows that the eigenvalues of (5.1) lie in a region close to this interval.

Theorem 5.2. For large enough $\nu$, the eigenvalues of the generalized problem (5.1) are contained in a region that is independent of the discretization mesh size $h$.

Proof. The Rayleigh quotient for the eigenvalue $\mu$ is

$$
\mu=\frac{\left(p,\left(S_{\infty}-\frac{1}{\nu} B A^{-1 / 2} \hat{N} A^{-1 / 2} B^{\mathrm{T}}+\frac{1}{\nu^{2}} B A^{-1 / 2} E A^{-1 / 2} B^{\mathrm{T}}\right) p\right)}{\left(p,\left(M_{p}-\frac{1}{\nu} M_{p}^{1 / 2} \hat{N}_{p} M_{p}^{1 / 2}+\frac{1}{\nu^{2}} M_{p}^{1 / 2} E_{p} M_{p}^{1 / 2}\right) p\right)} .
$$

Dividing numerator and denominator by $\left(p, M_{p} p\right)$, we rewrite this as

$$
\frac{\frac{\left(p, S_{\infty} p\right)}{\left(p, M_{p} p\right)}-\frac{1}{\nu} \frac{\left(p, B A^{-1 / 2} \hat{N} A^{-1 / 2} B^{\mathrm{T}} p\right)}{\left(p, M_{p} p\right)}+\frac{1}{\nu^{2}} \frac{\left(p, B A^{-1 / 2} E A^{-1 / 2} B^{\mathrm{T}} p\right)}{\left(p, M_{p} p\right)}}{1-\frac{1}{\nu} \frac{\left(p, M_{p}^{1 / 2} \hat{N}_{p} M_{p}^{1 / 2} p\right)}{\left(p, M_{p} p\right)}+\frac{1}{\nu^{2}} \frac{\left(p, M_{p}^{1 / 2} E_{p} M_{p}^{1 / 2} p\right)}{\left(p, M_{p} p\right)}} .
$$

Now consider the individual quotients appearing in (5.6). For the numerator, we have already observed that the first term is contained in $\left[\gamma^{2}, \Gamma^{2}\right]$. The second term is imaginary and satisfies

$$
\begin{aligned}
\left|\frac{\left(p, B A^{-1 / 2} \hat{N} A^{-1 / 2} B^{\mathrm{T}} p\right)}{\left(p, M_{p} p\right)}\right| & =\left|\frac{\left(p, B A^{-1 / 2} \hat{N} A^{-1 / 2} B^{\mathrm{T}} p\right)}{\left(p, S_{\infty} p\right)}\right| \frac{\left(p, S_{\infty} p\right)}{\left(p, M_{p} p\right)} \\
& \leq \rho(\hat{N}) \Gamma^{2} .
\end{aligned}
$$

Similarly, the third quotient is bounded by

$$
\left|\frac{\left(p, B A^{-1 / 2} E A^{-1 / 2} B^{\mathrm{T}} p\right)}{\left(p, M_{p} p\right)}\right| \leq \frac{\left\|A^{-1 / 2} B^{\mathrm{T}} p\right\|_{2}^{2}}{\left(p, M_{p} p\right)}\|E\|_{2} \leq \rho(\hat{N})^{2} \Gamma^{2} .
$$

Analogous arguments apply for the two quotients appearing in the denominator of (5.6). It follows that the eigenvalue $\mu$ must be of the form

$$
\frac{\alpha+i \beta}{\tau+i \eta}
$$

where $\alpha \in\left[\gamma^{2}-c \nu^{-2}, \Gamma^{2}+c \nu^{-2}\right], \tau \in\left[1-c \nu^{-2}, 1+c \nu^{-2}\right]$, and $\beta, \eta$ are real of order $\nu^{-1}$. 


\subsection{General results}

For the case of general $\nu$, we begin with the generalized eigenvalue problem

$$
\left(\begin{array}{cc}
F & B^{\mathrm{T}} \\
B & 0
\end{array}\right)\left(\begin{array}{l}
u \\
p
\end{array}\right)=\lambda\left(\begin{array}{cc}
F & 0 \\
0 & \hat{P}_{S}
\end{array}\right)\left(\begin{array}{l}
u \\
p
\end{array}\right),
$$

which arises from application of a block diagonal preconditioner to (1.4), as in $[4,20,23]$. It follows from the first equation of (5.7) that for $\lambda \neq 1$, $u=\left(\frac{1}{\lambda-1}\right) F^{-1} B^{\mathrm{T}} p$, and the problem (5.1) for the Schur complement can be derived by eliminating $u$ from the coupled system and setting $\mu=$ $\lambda(\lambda-1)$. Alternatively, the second equation of (5.7) gives $p=\frac{1}{\lambda} \hat{P}_{S}^{-1} B u$, and elimination of $p$ yields the generalized problem

$$
B^{\mathrm{T}} \hat{P}_{S}^{-1} B u=\mu F u \text {. }
$$

By analogy with terminology used in the optimization literature, we refer to (5.8) as the primal problem associated with (5.7); equation (5.1) is the related dual problem. The eigenvalues of (5.8) consist of the nonzero eigenvalues of (5.1) together with a zero eigenvalue of multiplicity equal to the dimension of the null space of $B$.

We will use the primal problem to derive a bound on the imaginary parts of these eigenvalues. Making the change of variables $u=A^{-1 / 2} v$ in (5.8) and premultiplying both sides by $A^{-1 / 2}$ leads to the equivalent problem

$$
A^{-1 / 2} B^{\mathrm{T}} M_{p}^{-1 / 2} A_{p}^{-1 / 2} F_{p} A_{p}^{-1 / 2} M_{p}^{-1 / 2} B A^{-1 / 2} v=\mu A^{-1 / 2} F A^{-1 / 2} v .
$$

We rewrite this in shorthand notation as

$$
(\nu J+K) v=\mu(\nu I+\hat{N}) v
$$

where $\hat{N}$ is as in Sect. 5.1 and

$$
\begin{aligned}
& J=A^{-1 / 2} B^{\mathrm{T}} M_{p}^{-1} B A^{-1 / 2}, \\
& K=A^{-1 / 2} B^{\mathrm{T}} M_{p}^{-1 / 2} A_{p}^{-1 / 2} N_{p} A_{p}^{-1 / 2} M_{p}^{-1 / 2} B A^{-1 / 2} .
\end{aligned}
$$

We have noted the boundedness of $\lambda_{\max }(\hat{N})$ and $\lambda_{\max }\left(\hat{N}_{p}\right)$ above. The following result establishes similar bounds on $J$ and $K$.

Lemma 5.3. The maximum eigenvalues of the matrices $J$ and $K$ of (5.10) are bounded in magnitude by constants that are independent of the parameters $h$ and $\nu$.

Proof. The assertion for $J$ follows from the boundedness of the maximum eigenvalue of the generalized problem (5.5). For a bound on the eigenvalues of $K$, we have

$$
\left|\lambda_{\max }(K)\right| \leq\left\|A^{-1 / 2} B^{\mathrm{T}} M_{p}^{-1 / 2}\right\|_{2}^{2}\left\|A_{p}^{-1 / 2} N_{p} A_{p}^{-1 / 2}\right\|_{2}
$$

The expression on the right is $\lambda_{\max }(J) \rho\left(\hat{N}_{p}\right)=O(1)$. 
That is, the matrices on each side of equation (5.9) consist of the sum of a bounded symmetric operator (times $\nu$ ) and a bounded skew-symmetric operator. A heuristic explanation for the effectiveness of the preconditioner is that, from (5.5), $J$ behaves loosely like an identity operator on the space range $\left(A^{-1 / 2} B^{\mathrm{T}}\right)$, and $K$ and $\hat{N}$ are derived in a similar manner from the underlying discrete convection operators. We also have the following result establishing a bound on the imaginary parts of the eigenvalues of the preconditioned system consistent with the behavior reported in Sect. 3.

Theorem 5.4. For small values of the viscosity parameter $\nu$, the maximum values of the imaginary parts of the eigenvalues of (5.1) are bounded by a quantity that grows like $O\left(\nu^{-1}\right)$ and is independent of the discretization parameter $h$.

Proof. From the equivalence of (5.1), (5.8) and (5.9), we seek a bound on the imaginary part of the Rayleigh quotient

$$
\mu=\frac{\nu(v, J v)+i s_{1}(v)}{\nu(v, v)+i s_{2}(v)},
$$

where $i s_{1}(v)=(v, K v), i s_{2}(v)=(v, \hat{N} v)$. This can be rewritten as

$$
\mu=\frac{\nu^{2}(v, J v)(v, v)+s_{1}(v) s_{2}(v)}{\nu^{2}(v, v)^{2}+s_{2}(v)^{2}}+i \frac{\nu\left[(v, v) s_{1}(v)-(v, J v) s_{2}(v)\right]}{\nu^{2}(v, v)^{2}+s_{2}(v)^{2}} .
$$

The imaginary part is bounded by

$$
\begin{aligned}
\Im(\mu) & \leq \frac{\nu\left[(v, v)\left|s_{1}(v)\right|+(v, J v)\left|s_{2}(v)\right|\right]}{\nu^{2}(v, v)^{2}+s_{2}(v)^{2}} \\
& \leq \frac{1}{\nu}\left(\left|\lambda_{\max }(K)\right|+\lambda_{\max }(J)\left|\lambda_{\max }(\hat{N})\right|\right) .
\end{aligned}
$$

\section{Full Picard iteration}

All the results above are for a fixed velocity field $\mathbf{w}$ in the Oseen equations (1.2) where, in the experiments, $\mathbf{w}$ was chosen to resemble the driven cavity flow field. The fixed wind was used for convenience but it is somewhat artificial, since the structure of the solution to (1.1) and of the iterates of (1.3) will depend on the viscosity $\nu$. In this section, we show the results of some experiments with the full nonlinear Picard iteration, for both discretizations and $h=1 / 64$.

Table 6.1 shows the average iteration counts required when preconditioned GMRES is used to solve each linear system arising during the nonlinear iteration. For completeness, the number of Picard iterations is also 
Table 6.1. Average inner iteration counts for Picard iteration, with outer iterations in parentheses, for $h=1 / 64$

\begin{tabular}{lccccc}
\hline & \multicolumn{5}{c}{$\nu$} \\
& $1 / 20$ & $1 / 40$ & $1 / 80$ & $1 / 160$ & $1 / 320$ \\
\hline MAC & $6.5(4)$ & $9.0(5)$ & $11.6(7)$ & $15.6(9)$ & $22.5(11)$ \\
$P_{2}-P_{1}$ & $6.8(4)$ & $8.3(4)$ & $9.6(5)$ & $13.2(6)$ & $18.1(7)$ \\
\hline
\end{tabular}

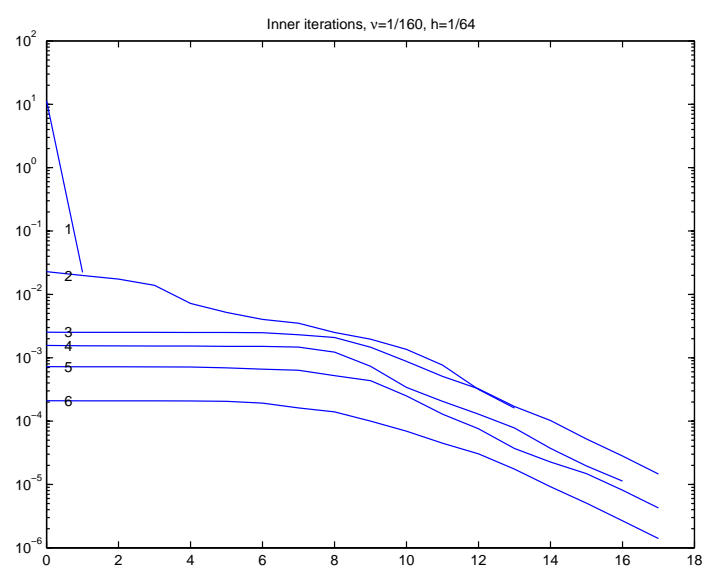

Fig. 6.1. Convergence histories of preconditioned GMRES inner iteration for the full Picard iteration with $\nu=1 / 160, P_{2}-P_{1}$ discretization, $h=1 / 64$

shown, in parentheses. These tests were run using an "inexact" nonlinear iteration (1.3), with the stopping criterion for the linear solver tied to the residual of the nonlinear system. That is, the linear iteration was stopped at step $k$ when the linear residual vector $r_{k}$ satisfied

$$
\left\|r_{k}\right\|_{2} \leq 10^{-2} \| F\left(\mathbf{x}^{(m-1)} \|_{2},\right.
$$

where $\mathbf{x}^{(m-1)}=\left(\begin{array}{l}\mathbf{u}^{(m-1)} \\ p^{(m-1)}\end{array}\right)$ and $F\left(\mathbf{x}^{(m-1)}\right)$ is the nonlinear residual. The starting iterates were $\mathbf{x}^{(0)} \equiv 0$ for the nonlinear system and the most recent nonlinear iterate for the linear system.

These results are consistent with what we observed for a fixed velocity field. In particular, the dependence on $\nu$ shown in Table 6.1 is essentially the same as that depicted in Table 2.1. Similar results are also given in [14]. The lower iteration counts are due to the less stringent stopping criterion. Further comparison is provided by Fig. 6.1, which shows the complete convergence histories of the linear solves for one problem, with $\nu=1 / 160$ and the $P_{2}-P_{1}$ discretization. This data is typical of the results for all the problems. Except in the first nonlinear step, which requires a Stokes solve, there is a latency exactly like that observed above (Fig. 2.2). 


\section{Concluding remarks}

The purpose of this study was to explore the properties of the preconditioning operator (1.5) proposed by Kay and Loghin, and to explain its behavior for the steady-state Navier-Stokes equation. Our results indicate that the eigenvalues of the preconditioned operator determine the convergence properties of preconditioned GMRES. Eigenvalue distributions and convergence rates are independent of discretization mesh size, and a small, $\nu$-dependent number of outlying eigenvalues lead to some dependence of performance on $\nu^{-1}$.

We conclude with a few comments on issues not explored here. First, we have not considered algorithmic costs. An iterative solver using this preconditioner can be constructed with building blocks consisting of a convectiondiffusion solver (for $P_{F}^{-1}$ ), a Poisson solver (for $A_{p}^{-1}$ ), a solver for the pressure mass matrix (i.e., for $M_{p}^{-1}$ ), and various matrix products. The latter two operations have negligable costs, and if fast algorithms such as multigrid or domain decomposition are used for the other tasks, then the cost per step of the complete iteration will be low. Kay and Loghin [14] showed that use of such inner iterations leads to performance consistent with that of the "exact" preconditioner. In addition, although we have restricted our attention to GMRES for the Krylov subspace method, we expect other solvers to perform well also.

Finally, this study has been limited to the steady-state problem. For evolutionary problems, the same solution algorithm can be applied at each step of a time-implicit iteration. Results in [22] suggest that in this setting, for small enough time steps, iteration counts appear to be independent of the viscosity.

\section{References}

1. D. N. Arnold, R. S. Falk, R. Winther: Preconditioning in H(div) and applications. Math. Comp. 27, 957-984 (1997)

2. J. H. Bramble, J. E. Pasciak: A preconditioning technique for indefinite systems resulting from mixed approximations of elliptic problems. Math. Comp. 50, 1-17 (1988)

3. E. Dean, R. Glowinski: On some finite element methods for the numerical simulation of incompressible viscous flow. In M. D. Gunzburger, R. Y. Nicolaides (ed.) Incompressible Computational Fluid Dynamics, pp. 17-65. New York: Cambridge University Press 1993

4. H. Elman, D. Silvester: Fast nonsymmetric iterations and preconditioning for NavierStokes equations. SIAM J. Sci. Comput. 17, 33-46 (1996)

5. H. C. Elman: Preconditioning for the steady-state Navier-Stokes equations with low viscosity. SIAM J. Sci. Comput. 20, 1299-1316 (1999)

6. H. C. Elman, M. H. Schultz: Preconditioning by fast direct methods for nonselfadjoint nonseparable elliptic problems. SIAM J. Numer. Anal 23, 44-57 (1986) 
7. B. Fischer, A. Ramage, D. Silvester, A. J. Wathen: Minimum residual methods for augmented systems. BIT 38, 527-543 (1998)

8. R. W. Freund: Quasi-kernel polynomials and their use in non-Hermitian matrix iterations. J. Comput. Appl. Math. 43, 135-158-869 (1992)

9. R. W. Freund, N. M. Nachtigal: A new Krylov-subspace method for symmetric indefinite linear systems. In W. F. Ames, editor, Proceedings of the 14th IMACS World Congress on Computational and Applied Mathematics, pp. 1253-1256. IMACS, 1994

10. V. Girault, P. A. Raviart: Finite Element Approximation of the Navier-Stokes Equations. New York: Springer 1986

11. F. H. Harlow, J. E. Welch: Numerical calculation of time-dependent viscous incompressible flow of fluid with free surface. The Physics of Fluids 8, 2182-2189 (1965)

12. A. Jennings: Influence of the eigenvalue spectrum on the convergence rate of the conjugate gradient method. J. Inst. Maths. Applics. 20, 61-72 (1977)

13. O. A. Karakashian: On a Galerkin-Lagrange multiplier method for the stationary Navier-Stokes equations. SIAM. J. Numer. Anal. 19, 909-923 (1982)

14. D. Kay, D. Loghin: A Green's function preconditioner for the steady-state NavierStokes equations. Technical Report 99/06, Oxford University Computing Laboratory, 1999

15. A. Klawonn: Block-triangular preconditioners for saddle point problems with a penalty term. SIAM. J. Sci. Comput. 19, 172-184 (1998)

16. M. F. Murphy, G. H. Golub, A. J. Wathen: A note on preconditioning for indefinite linear systems. SIAM J. Sci. Comput. 21, 1969-1972 (2000)

17. R. A. Nicolaides: Analysis and convergence of the MAC scheme I. SIAM J. Numer. Anal. 29, 1579-1591 (1992)

18. R. A. Nicolaides, X. Wu: Analysis and convergence of the MAC scheme. II. NavierStokes equations. Math. Comput. 53, 29-44 (1996)

19. T. Rivlin: Chebyshev Polynomials: From Approximation Theory to Algebra and Number Theory. New York: John Wiley \& Sons 1990

20. T. Rusten, R. Winther: A preconditioned iterative method for saddle point problems. SIAM J. Matr. Anal. Appl. 13, 887-904 (1992)

21. Y. Saad, M. H. Schultz: GMRES: A generalized minimal residual algorithm for solving nonsymmetric linear systems. SIAM J. Sci. Stat. Comput. 7, 856-869 (1986)

22. D. Silvester, H. Elman, D. Kay, A. Wathen: Efficient Preconditioning of the Linearized Navier-Stokes Equations for Incompressible Flow. Technical Report UMIACS-TR-9966, Institute for Advanced Computer Studies, University of Maryland, 1999. J. Comp. Appl. Math. 128, 261-279 (2001)

23. D. Silvester, A. Wathen: Fast iterative solution of stabilized Stokes systems. Part II: Using block preconditioners. SIAM J. Numer. Anal. 31, 1352-1367 (1994)

24. G. Stoyan: Towards discrete Velte decompositions and narrow bounds for inf-sup constants. Computers \& Math. Applics. 38, 243-261 (1999)

25. S. Turek: Efficient Solvers for Incompressible Flow Problems. Berlin: Springer, 1999

26. A. Wathen, D. Silvester: Fast iterative solution of stabilized Stokes systems. Part I: Using simple diagonal preconditioners. SIAM J. Numer. Anal. 30, 630-649 (1993) 